\title{
Characterisation of the high strain rate behaviour of tubular materials
}

\author{
Camille Caisso ${ }^{1,2,}$, , Nicolas Jacques ${ }^{1}$, Aboulghit El Malki Alaoui ${ }^{1}$, Harold Fresnel $^{2}$ and Younes Demmouche ${ }^{1}$ \\ ${ }^{1}$ Institut de Recherche Dupuy de Lôme, FRE CNRS 3744, ENSTA Bretagne, 29200, Brest, France \\ ${ }^{2}$ Autoliv Livbag, Route du Beuzit, 29590, Pont-de-Buis, France
}

\begin{abstract}
Airbag gas generators used in the automotive industry are often fabricated from tubular materials. The present work deals with the development of split Hopkinson tensile bar (SHTB) tests for tubular samples. Tubular specimens were machined from the tubes. A specific gripping system was designed to install the samples between the incident and transmitter bars. The forces acting on the samplegrip assembly were measured using strain gages mounted on the bars. Strain gages were also placed on the specimen in order to obtain the local strain history. Finite element computations were carried out to assess the validity of the experimental setup. It was observed that, in some cases, a vibration of the gripping system may induce oscillations on the force signals. To limit this phenomenon, pulse shapers [1] were employed in order to obtain a smoother input wave. Several tests were performed for different impact velocities. Strain rates ranging between 100 and $700 \mathrm{~s}^{-1}$ were achieved. Quasi-static tensile tests were also carried out. From the results of the different experiments, it was found that the steel under investigation has significant strain-rate sensitivity. Using inverse analysis, parameters for the Zerilli-Armstrong constitutive model [2] were identified.
\end{abstract}

\section{Introduction}

This study takes place in airbag gas generator industry, where mass reduction and robustness are critical quality factors. During operation, the material of gas generator chambers is subjected to severe dynamic loading conditions. Consequently, the design of gas generator requires the characterisation of the High strain-behaviour of their constitutive material. However as, gas generators are produced from thin walled tube, material characterisation requires the development of specific tests.

Different experimental set-ups have been developed to characterize the quasi-static elastic-plastic behaviour of tubular materials [3]. Standard testing machines allow achieving strain-rates close to $1 \mathrm{~s}^{-1}$. To reach higher strain-rates, it is necessary to use high-speed testing machines or split Hopkinson pressure bar (SHPB) systems. Using split Hopkinson pressure bar, strain-rates to $1000 \mathrm{~s}^{-1}$ can be achieved. To our best knowledge, SHPB tensile tests applied to tubular materials were not described in bibliography.

The purpose of the present work is the development of an experimental SHPB setup adapted to tubular materials and of a methodology to characterise the high strain-rate behaviour of this kind of materials.

Finite element analyses were used to design the sample and the gripping system. Parameters such as gauge length, sample thickness and grips geometry were studied. The proposed set-up can be adapted on standard Hopkinson tensile bar systems.

Experiments were carried out. The obtained results are presented and analysed. A methodology is proposed to identify constitutive model parameters from the experimental measurements using finite element simulation and inverse analyse.

\section{Low strain-rate tensile tests}

The material considered in the present study is a lowcarbon high-strength steel. The production process involves operations including hot rolling, quenching, tempering and cold drawing. This process generates a bainitic microstructure with a small grain size.

The low strain rate behaviour of this material was characterised using quasi-static tensile tests. The samples were machined from tubes. Several studies have shown that low carbon steels are sensitive to strain-rate [4-5]. In the present study, the tensile tests were carried out for two different strain-rates: $10^{-3} \mathrm{~s}^{-1}$ and $10^{-1} \mathrm{~s}^{-1}$. Tests with a strain-rate jump were also performed. The force and strain history were recorded by the testing machine load sensor and by an Epsilon ${ }^{\mathrm{TM}}$ uniaxial extensometer, respectively.

\footnotetext{
Corresponding author: camille.caisso@ensta-bretagne.org
} 


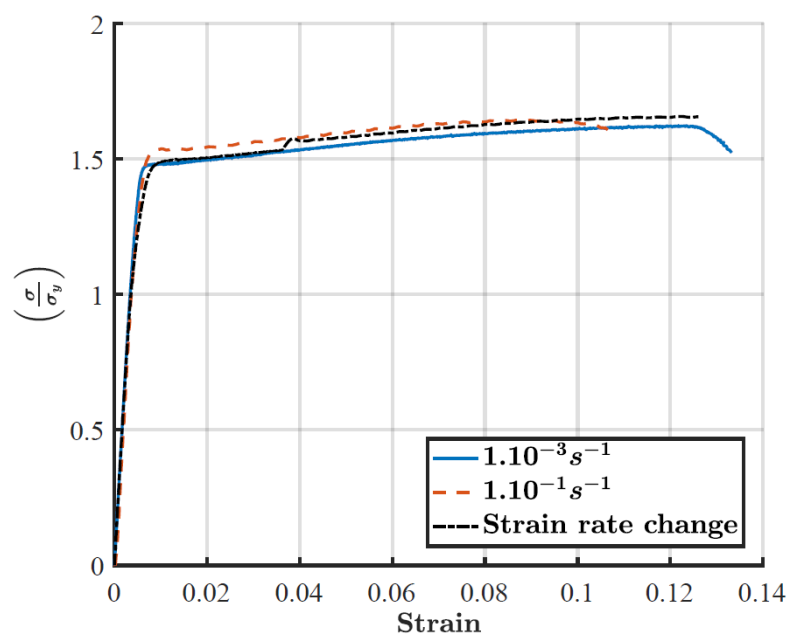

Fig. 1. Quasi-static stress-strain curves of the considered steel (the curves have been normalized using the initial yield stress of the material). Two strain-rates were considered: $10^{-3} \mathrm{~s}^{-1}$ and $10^{-1} \mathrm{~s}^{-1}$. The results of a test involving a strain-rate jump from $10^{-3} \mathrm{~s}^{-1}$ to $10^{-1} \mathrm{~s}^{-1}$ are also presented.

Some stress-strain curves obtained with the quasistatic tests are shown in Fig.1. The present material presents a very low strain-hardening. Moreover, it can be observed that, even under quasi-static conditions, the considered steel exhibits some strain-rate dependency. The quasi-static tests enabled to identify the Young modulus and the hardening curve of the material.

\section{High strain-rate experiments}

\subsection{Samples and gripping system}

Cylindrical dog-bone-shaped specimens commonly used in Hopkinson bar tensile tests [6] are not applicable in the present case because of the tubular geometry of the base material. Thus, thin-walled cylindrical specimens have been employed. A complementary gripping system $[4,7,8]$ has been developed. It enforces a mechanical connection between the sample, the incident bar and the transmitter bar, see Fig. 2.

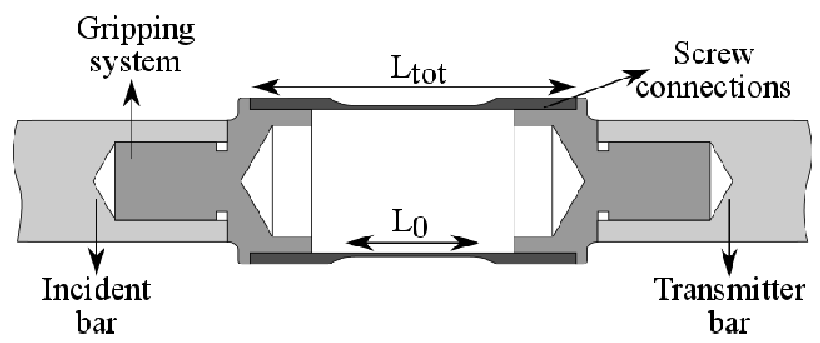

Fig. 2. Proposed sample geometry and gripping system.

The diameter of the samples is imposed by the initial tube dimensions. For this reason, the samples have a diameter of $30 \mathrm{~mm}$ that is larger than the diameter of the bars $(24 \mathrm{~mm})$. In the gauge section, the thickness is reduced from $2 \mathrm{~mm}$ to $0.7 \mathrm{~mm}$. In addition, to avoid failure risks close to sample threads, a very thin pitch is used.
Using finite element simulations (see section 5.2), it was observed that the specimen gauge length and the impact velocity control the final strain level (Fig. 3). A sample gauge length of $20 \mathrm{~mm}$ was adopted. This value leads to a final strain level of about 5\% and a maximum strain-rate close to $700 \mathrm{~s}^{-1}$. A drawback of using a relatively short length is that the strain distribution is not homogeneous within the specimen. To circumvent this problem, a local measure of the strain at the centre of the specimens was performed during the tests using strain gauges. It should be noticed that the stress in the gauge section is almost uniaxial (the triaxiality factor is of $0.332)$

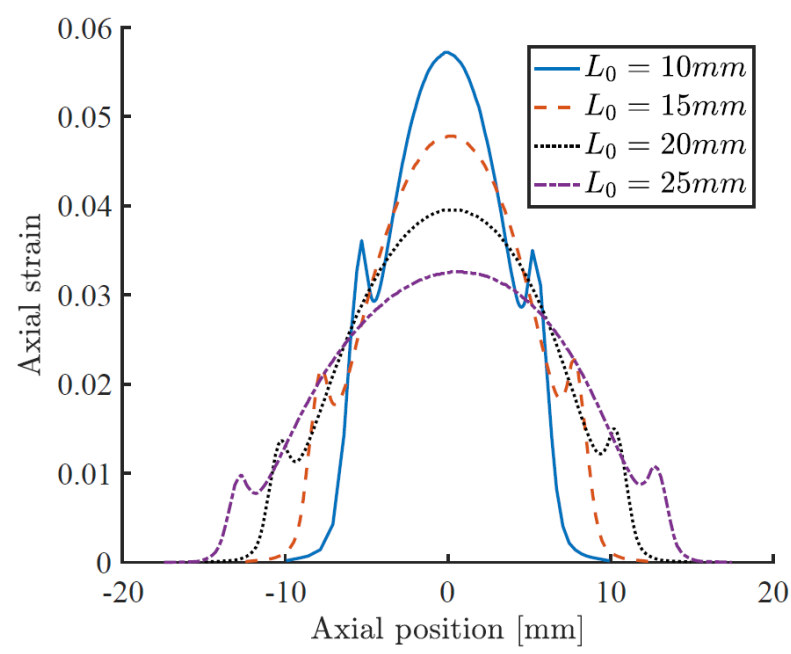

Fig. 3. Strain distribution within the specimen for different sample gauge lengths for an impact velocity of $18.1 \mathrm{~m} / \mathrm{s}$ (from finite element simulations).

For testing metallic materials, usually screw connections are used to avoid any possible slip between the components. A second advantage of this technique is to limit backlash. Fixtures are made of steel having similar mechanical properties (in particular a mechanical impedance) than the bar material. Several fixture designs were tested with the finite element simulations to minimise the mass fixtures and limit inertia effects. However, for some loading conditions, the system (sample + grip) doesn't reach static equilibrium during the test. To limit this problem, pulse shapers have been employed. This point will be discussed in section 4.3.

Alignment of the experimental is a key point of tensile tests on tubular material. Induced bending of bars or sample should be avoided. This was achieving by restrictive tolerances on fixtures and samples.

\subsection{Experimental set-up}

The high strain-rate tests were performed with the SHPB system of the Institute of Research Dupuy de Lôme. The bars are made of maraging steel and have a diameter of $24 \mathrm{~mm}$. The tensile stress wave is generated by the impact of a striker tube that travels along the incident bar. The striker is propelled with a gas gun.

Signals are registered with strain gauges mounted on the incident and transmitter bars at a distance of $1 \mathrm{~m}$ 
from the sample. Gauges are wired into a Wheatstone bridge to cancel temperature effects.

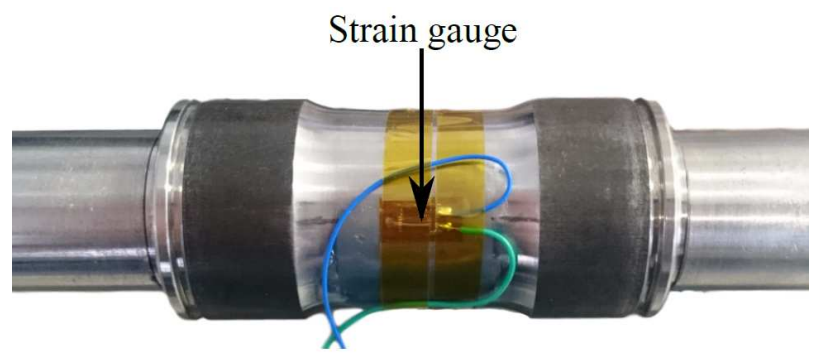

Fig. 4. Experimental set-up for the high strain rates tensile tests.

The sample strain is measured with two strain gauges located at diametrically opposed positions, Fig.4. Thereby, possible bending effects can be eliminated by considering the mean value of the recordings of the two gauges. A HBM Data acquisition system Geni3 was used with a sampling frequency of $1 \mathrm{MHz}$.

\subsection{Pulse shaping and incident wave signals}

The results of Hopkinson bar tests are dependent of the shape of the incident stress pulse. With our SHPB, the stress waves generated have a square shape with oscillations due to radial inertia (Pochammer-Chree oscillations [6]) and probably to electro-magnetic perturbations, that may occurs with steel bars [9-10], see Fig. 5. From finite element simulations, we observed that the reflection of a square pulse at the junction between the incident bar and the grip causes a large force peak, which could damage the threads. Consequently, the use of pulse shapers [1, 11-14] has been considered, with following aims:

-To attenuate signal oscillations

-To increase rise time and incident pulse duration

-To promote sample equilibrium

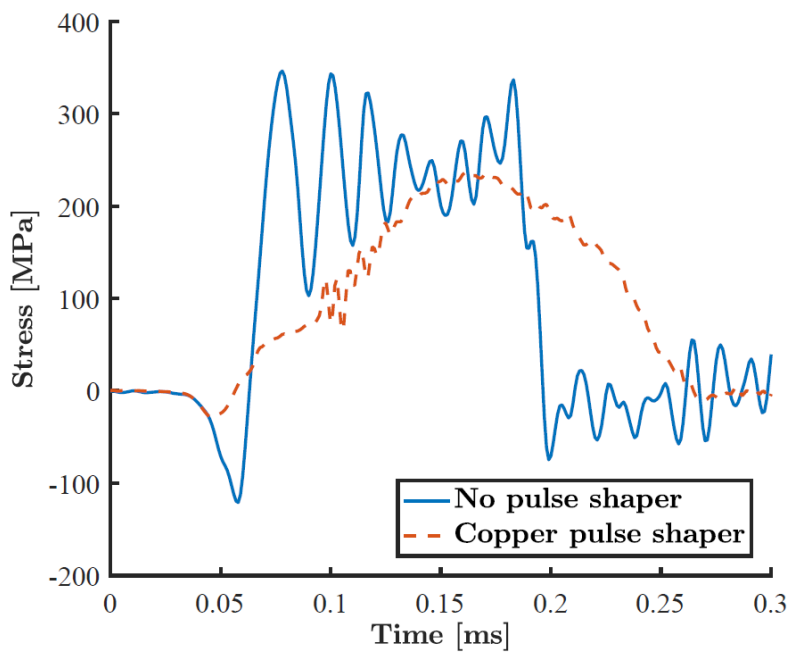

Fig. 5. Comparison of incident signals obtained with and without pulse shaper.

As our SHPB device uses a projectile impacting a flange, the proposed solution was to place a dummy piece (pulse shaper) on the impact surface of the flange, see Fig. 6. Several kinds of pulse shapers were tested: $1 \mathrm{~mm}$ thick rubber sheets, single spire copper wires, double spire copper wires. The best results, in terms of repeatability, shape and smoothness of the incident stress signal, were obtained with single spire copper wires (Fig. 7 ). The high ductility of copper and the cylindrical wire leads to a gradual stress rise and to the suppression of oscillations. This pulse shaping technique was employed in all tests.

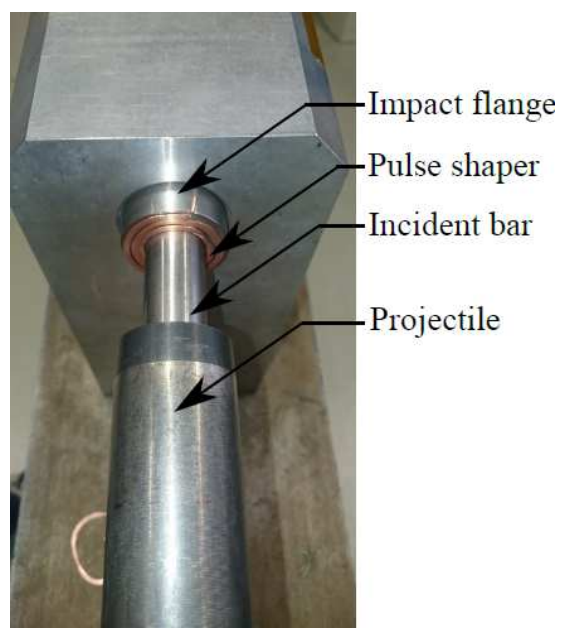

Fig. 6. Pulse shaping technique. A copper wire is placed on the impact surface of the incident bar flange.

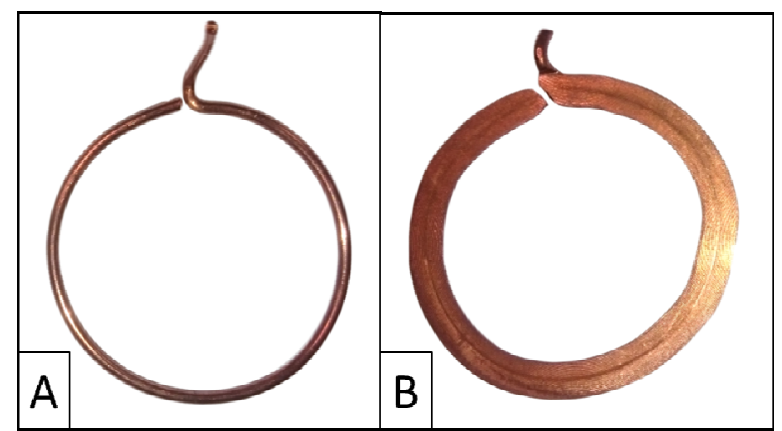

Fig. 7. Single spire copper wire pulse shaper. (A) initial geometry; (B) shape after impact.

\subsection{Experimental results}

Tests have been carried out at ambient temperature for different impact velocities ranging from 11.5 to $24 \mathrm{~m} / \mathrm{s}$ leading to peak strain-rates ranging between 100 and 800 $\mathrm{s}^{-1}$. Typical stress-strain curves derived from the SHPB tests are presented in Fig. 8 and compared to the static response of the material. It is reminded that the strain history is measured directly in the specimen using strain gauges. The stress history is deduced from the signal recorded on the transmitter bar. The propagation delay between sample and transmitted bar was determined from the experimental signals considering the stage where the specimen material deforms elastically [15]. 


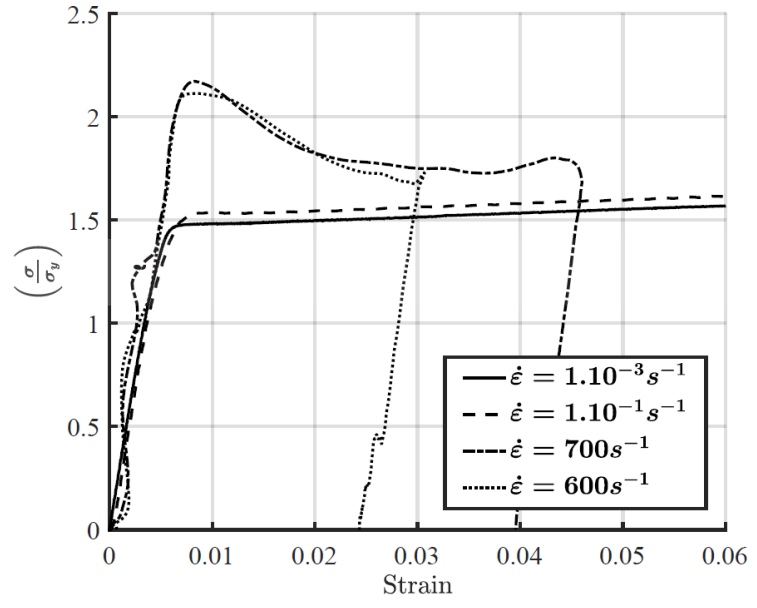

Fig. 8. Stress-strain curves obtained from quasi-static tensile tests and SHPB tests (the curves have been normalized using the initial yield stress of the material). It should be noted that during SHPB tests, the strain-rate is not constant; the values indicated in the legend correspond to the peak value.

From Fig. 8, it can be observed that high strain-rate has a noticeable effect on the material response with a significant rise of the stress level. It also appears that the shape of the stress-strain curves derived from the Hopkinson tests is different from the quasi-static curves. The yield stress reaches its maximum value at the end of the elastic stage, followed by a decrease of the stress. This phenomenon cannot be related to the strain-rate history, as the maximum strain-rate is achieved latter (Fig. 9). Using finite element simulations, we observed this phenomenon is due to a vibration of the gripping system. This is illustrated by the Fig. 10 that displayed the stress history within the specimen and the stress deduced from the transmitter bar recording. A similar behaviour was observed by Qin et al. [16]. They showed that, for materials with an abrupt elastic-plastic transition (materials with low strain hardening or with a yield point), an oscillation of the gripping system may take place and cause perturbations of the force measurements.

Because of this ringing phenomenon, a direct analysis of the tests cannot be done and to identify parameters of material models, it is necessary to resort to numerical simulations.

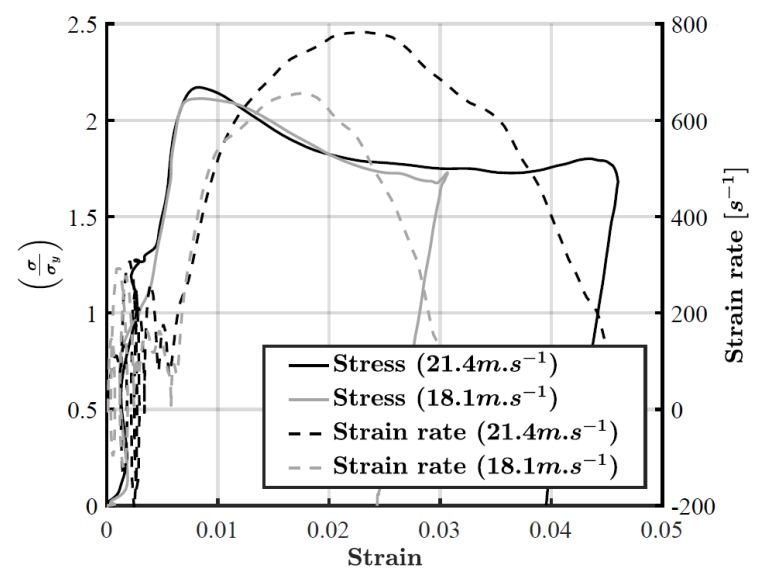

Fig. 9. Strain-rate histories associated to stress-strain curves obtained for two impact velocities (18.1 and $21.4 \mathrm{~m} / \mathrm{s}$ ).

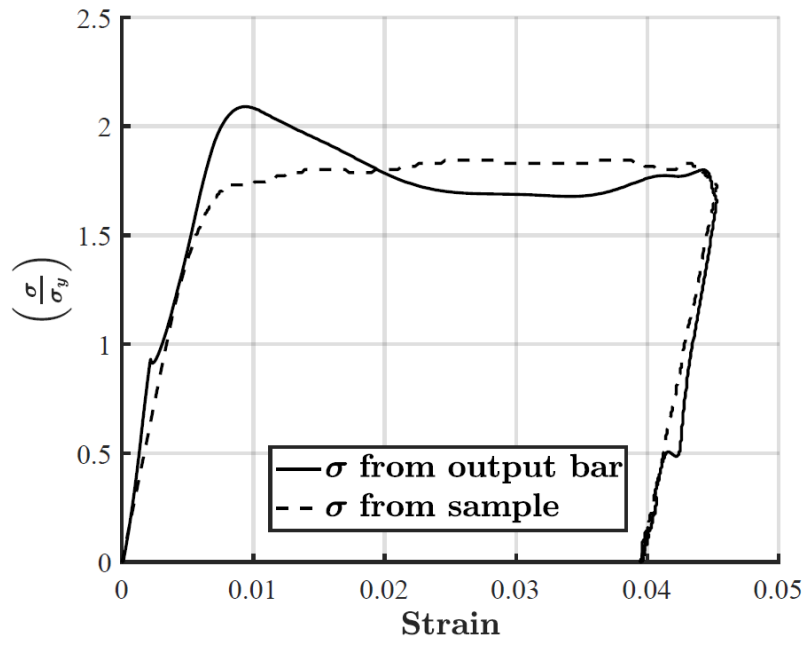

Fig. 10. Comparison between the stress history at centre of the specimen and the stress history derived from the signal recorded on the transmitter bar. Results of a finite element simulation.

\section{Identification of Zerilli-Armstrong model parameters}

\subsection{Zerilli-Armstrong constitutive model}

Zerilli and Armstrong [2] proposed dislocationmechanics-based constitutive relations for $\mathrm{FCC}$ and $\mathrm{BCC}$ metals. In the case of BCC metals, the flow stress of the material is written as the sum of an athermal stress $\sigma_{y}$ (that depends on strain) and of a thermally-activated stress $\sigma^{*}$ (that depends on strain-rate and temperature):

$$
\sigma=\sigma_{y(p)}+\sigma^{*}(p, \dot{T})
$$

with,

$$
\sigma^{*}(\dot{p}, T)=C_{1} * \exp \left(-C_{2} * T+C_{3} * T * \ln (\dot{p})\right)
$$

It should be noted that, for isothermal conditions, the expression of the thermally-activated stress can be rewritten as:

$$
\sigma^{*}(\dot{p}, T)=C_{1} \approx \exp \left(m * \ln \left(\frac{\dot{p}}{D_{0}}\right)\right) ;
$$

where $C_{l}, m$ and $D_{0}$ are material parameters that characterises the strain-rate sensitivity of the material. The ZA model is based on the idea that dislocation motion is a thermally-activated process. During plastic deformation, dislocations have to overcome different types of obstacles, generally classified into long range and short range. Only short range barrier can be overcome by thermal energy. In Eq. (1), the athermal stress is associated to long-range barriers and the thermally-activated stress to short-range barriers. More details about the physics of dislocation motion can be found in [17]. 


\subsection{Finite element model}

Finite element analyses were carried out for the geometry show in Fig. 2 using the non-linear finite element software ABAQUS/Explicit. An axisymmetric simplified model of the SHPB apparatus was developed. Only a part of the incident and transmitter bars are modelled and the striker and anvil are not considered. Typical incident wave signals, recorded on the experimental apparatus, are prescribed at the end of the incident bar. Moreover, in order to avoid fictions wave reflexions, non-reflecting boundary conditions are applied at the remote ends of the bars. The interactions between the different parts are described using a "mesh tie constraint", which prevents any relative displacement between the surfaces.

The bars and the grips have an elastic behaviour. The sample behaviour is described by the Zerilli-Armstrong model (implemented in ABAQUS using a VUMAT subroutine).

\subsection{Identification methodology}

An identification methodology was developed to determine constitutive model parameters using finite element analyses of the SHPB tests. It should be noted that the evolution of the athermal (rate-independent stress) was determined from quasi-static tensile tests. Therefore, we have to determine only the parameters related to the thermally-activated stress. It should also be noted that parameters $C_{l}$ and $D_{0}$ are not independent. Therefore, $D_{0}$ was arbitrary set to $10^{8} \mathrm{~s}^{-1}$. The values of $C_{I}$ and $m$ were determined using the finite element simulations. Several simulations were carried out for several values of $C_{l}$ and $m$, and we selected the set of parameters that leads to the better agreement with the experimental measurements. We considered both the stress history in the transmitter bar and the strain history in the specimen. The following of values of the parameters were obtained: $C_{1}=1903 \mathrm{MPa}, D_{0}=10^{8} \mathrm{~s}^{-1}$ and $m=0.1747$.

Fig. 11 presents a comparison between experimental measurements and numerical simulations (for the identified set of material parameters). A fairly good agreement is observed.

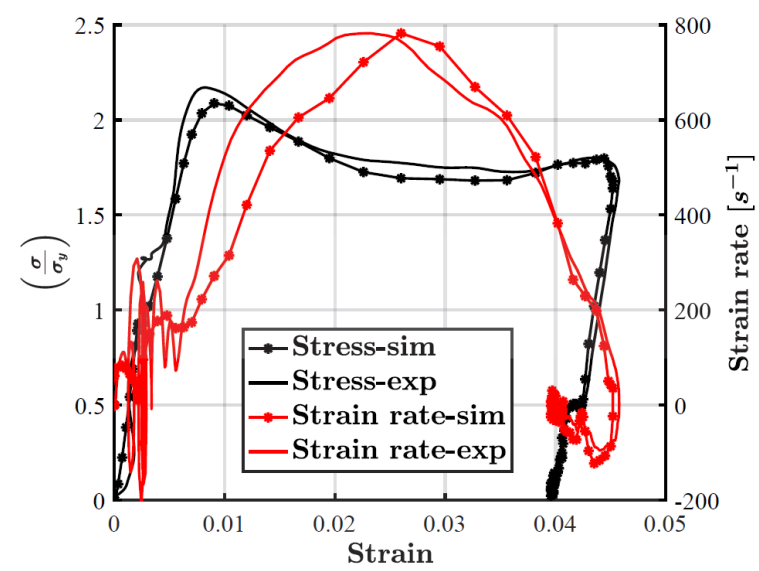

Fig. 11. Comparison between the experimental results and the results of a finite element simulation performed with the identified Zerilli-Armstrong models.

\section{Conclusions}

The present work deals with the characterisation of the high strain-rate behaviour of tubular materials. For this kind of material, it is not possible to use standard specimen. For this reason, we have developed a tubular specimen and a specific gripping system that can be used with Hopkinson tensile bar systems.

The proposed set-up has been applied to investigate the high strain-rate behaviour of a high-strength steel. Strain-rates up to $700 \mathrm{~s}^{-1}$ were achieved. Because this investigated material exhibits an abrupt elastic-plastic transition, a ringing of the gripping system was observed (this phenomenon is not likely to occur with materials having a more pronounced strain hardening). Therefore, to identify material parameters from the experimental recording, finite element simulations have been employed.

Authors wish to thank Mannesmann Precision Tubes France SAS for providing steel tubes, Autoliv Livbag and the French National Agency for Research and Technology (ANRT) for financial support.

\section{References}

1. R. Gerlach, S. Sathianathan, N. Petrinic, Int. J. of Impact Eng., 38, pp. 976-980 (2011)

2. F. Zerilli, R.W. Armstrong, J. Appl. Phys, 61, pp. 1816-1825 (1987)

3. H. Fresnel, V. Grolleau, P. Longère, G. Rio, P. Hardy, Thin-Walled Struct., 47, pp. 295-303 (2009)

4. V. Tarigopula, O.S. Hopperstad, M. Langseth, A.H. Clausen, F. Hild, Int. J. of Solids and Struct., 45, pp. 601-619 (2008)

5. B. Tanguy, J. Besson, R. Piques, A. Pineau, Eng. Frac. Mech., 72, pp. 49-72 (2005)

6. Y. Chen, A.H Clausen, O.S. Hopporstad, M. Langseth, Int. J. of Imp. Eng., 38, pp. 824-836 (2011)

7. T. Gomez-del Rio, E. Barbero, R. Zaera, C. Navarro, Comp. Sci. and Tech., 65, pp. 61-71 (2005)

8. K.H. Nguyen, H.C. Kim, H. Shin, Y. Yoo, J.B. Kim, Int. J. of Imp. Eng., 109, pp. 253-263 (2017)

9. A.D. Barr, S.D. Clarke, A. Tyas, J.A. Warren, Exp. Mech., 57, pp. 813-817 (2017)

10. A.H. Metzler, The Rew. Os Scien. Instr., 27 (1956)

11. R. Gerlach, C Kettenbeil, N. Petrinic, Int. J. of Imp. Eng., 50, pp. 63-67 (2012)

12. M. Hassan, K. Wille, Const. and Build. Mat., 144, pp. 747-757 (2017)

13. A.B. Shemirani, R. Naghdabadi, M.J. Ashrafi, Const. and Build. Mat., 125, pp. 326-336 (2016)

14. R. Naghdabadi, M.J Ashrafi, J. Arghavani, Mat. Sc. And Eng., 539, pp.285-293 (2012)

15. H. Zhao, Comp. \& Struct., 81, pp.1301-1310 (2003)

16. Z. Qin, J. Zhu, W. Li, Y. Xia, Q. Zhou, Int. J. of Imp. Eng., 108, pp. 295-302 (2017) 
17. M. M. Gurusamy, B. C. Rao, J. of Manu. Proc, 28, pp.253-265 (2017) 